\title{
TRANSPARENT ELECTRONICS: AN EMERGING FIELD IN TECHNOLOGY
}

\author{
Anushka Srivastava ${ }^{1}$ \\ ${ }^{I}$ Electronics and Communication Engineering Graduate, Institute of Technology, GIDA, Gorakhpur, Uttar Pradesh, \\ India
}

\begin{abstract}
Transparent Electronics, as a field of science and technology, has come into view and is increasing with huge leaps and bounds. It is trying to produce an entirely invisible electronic circuitry and opto-electronic devices. The first example of transparent electronics was reported by researchers at Oregon State University and Hewlett Packard to make transparent transistors that are inexpensive, stable and environment friendly. The Swiss Federal Institute of Technology made a transistor so thin that it can wrap around a human hair. Transparent electronics could be applied in various consumer electronics, new energy sources and transportation. For example, windshields of automobile which could transmit visual information to the driver, flexible electronics that could be folded up for ease of transport. When deposited onto glass, could double as an electronic device, possibly improving security systems or offering transparent displays. In the similar way, windows could be used to produce electrical power. The key component in these applications are wide band gap semiconductors, where oxides of different origin play an important role, not only as passive component but also as an active component similar to what we observe in conventional semiconductors with crystalline or amorphous like structure. The first scientific goal of this technology must be to discover, understand, and implement transparent high-performance electronic materials. Consequently, in order to enable this revolutionary technology requires bringing together expertise from various pure and applied sciences, including material science, chemistry, physics, electrical/electronic engineering, and display science.
\end{abstract}

Keywords: - Flexible electronics, invisible, transparent electronics, transparent transistors, wide band gap semiconductors. ****

\section{INTRODUCTION}

The world has been provided with 'transparency' and 'clarity' by the researchers. This clarity or transparency is possible only through 'transparent electronics'. The things which were only in the imaginative world, viz. windscreen of the car blinking to show our important messages, as in [1]; windows in the house controlling the light entering our room and finally making it dark, when required; windshields of the car showing the direction to reach a particular place, to the drivers; big solar panels becoming invisible; the computer systems, flexible and transparent enough to be transported with much ease; the light falling on the windows of the house could be utilised to generate electricity, as in [2],etc., has turned out to be in the real world.

It has been made possible only by the researchers, especially at Oregon State University (OSU) and Hewlett Packard (HP), who first reported about the transparent electronics. Then, the Swiss Federal Institute of Technology (SFIT) invented a transistor, thin enough to be wrapped around a human hair and to be put on the top of a contact lens, as in [3]. Figure 1 show an ultra-thin circuit, which is laid on the top of a contact lens by SFIT.

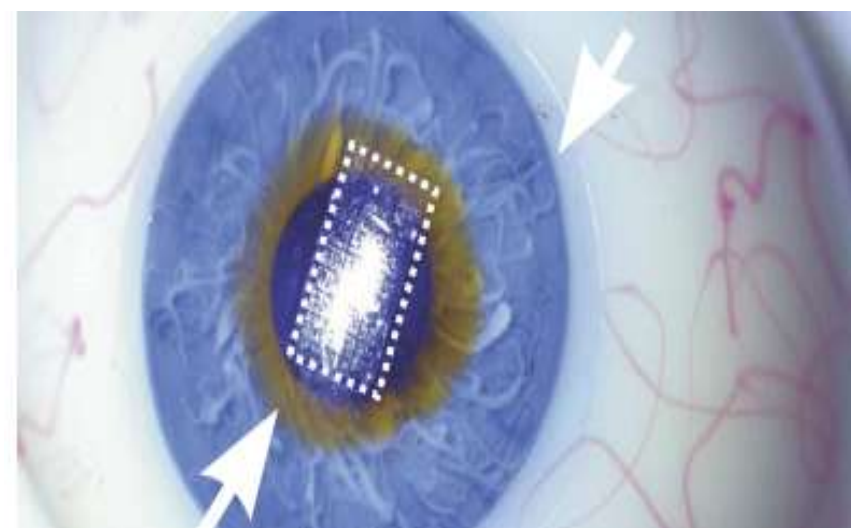

Fig 1: Swiss Federal Institute of Technology laid an ultrathin circuit on the top of a contact lens

Scientists of the Korean Advanced Institute of Science and Technology, Jung Won Seo, Jae-Woo Park, Keong Su Lim, Ji-Hwan Yang and Sang Jung Kang invented the world's first transparent computer chip, known as TRRAM or Transparent Resistive Random Access Memory which is a new milestone in transparent electronics. It can turn the windows or mirrors into computer monitors or television screens, as in [4]. Using TRRAM, transparent, flexible phones can be realized, as in [5]. Figure 2 shows the transparent smartphone. 


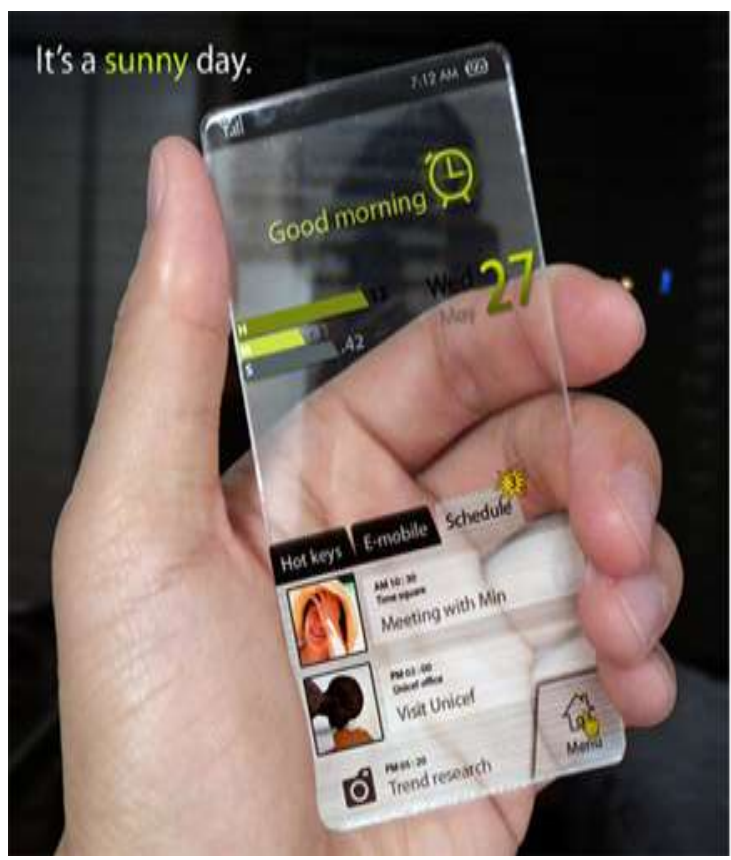

Fig 2: Transparent Smartphone

\section{MATERIALS AND METHODS}

The properties of making a transparent conductor as transparent, makes it an insulator, because 'transparency' and 'conductivity' are two contradictory properties. Yet, in the presence of this contradiction, zinc oxide came out with the success of researchers at OSU in 2003, as in [6]. Since then, researchers have found more than 28 different new material compounds, including indium tin oxide (ITO), which can all render transparent electronic circuits. Since the percentage of the constituents mixed can vary, Wager says that the new material possibilities are actually infinite in number.

The balance established between transparency and conductivity, in the transparent conducting oxides (TCO), has been made possible only by the presence of wide band gaps in them. Excitation of electrons takes place across these wide band gaps thus preventing the absorption of visible light and making them transparent, according to researchers, as in [7]. The dopant atoms, at the same time, increase the number of electrons by providing some additional electrons in the conduction band which as a result enables the electrical conductivity of the material. The free electrons generated, can also absorb light and consequently excited to higher conduction band states. Thus, transmission of visible light, contributes transparency while absorption of infrared and ultraviolet rays, contributes in electricity generation (when thin conducting film is attached to the window panes). Figure 3 shows a transparent conducting oxide layer, which is transmitting visible light and absorbing ultraviolet rays.

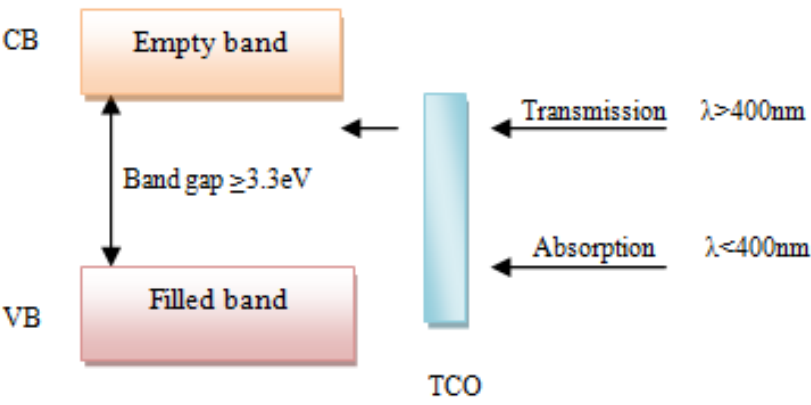

Fig 3: Transmission of visible light and absorption of UV rays by transparent conducting oxides

Apart from this, for developing the circuit, researchers needed a polymer which was thin and transparent but tough enough for the circuit to be etched into it. They even required a secondary polymer which could act as the support and at the same time, such that it dissolves in water after the circuitry was complete. Finally, parylene was chosen as the primary polymer and vinyl as the secondary polymer.

Parylene sometimes acts as a moisture and environmental barrier for rubber, plastic and electronic goods. It is biocompatible and very stable.

\section{RESULTS}

A thin sheet, whose width is roughly one-sixtieth the width of a human hair, alongwith the electronic components, could be obtained when parylene is adhered to vinyl. When the circuitry is etched, vinyl dissolves in water, as in [3]. The thin sheet obtained, is unbreakable and can be wrapped around a human hair. The researchers were successful in attaching the film to a contact lens and inventing a device that could monitor the eye pressure of the patient suffering from glaucoma. The electronic circuits, made by amorphous non-crystalline materials, reduces the production cost, as, the new materials could be printed on plastic substrates at very low temperatures.

Transparent thin film resistors (TTFR) and transparent thin film capacitors (TTFC), when properly designed, exhibit linear behaviour and can be modelled as an ideal resistor and an ideal capacitor, respectively. Unlike TTFR and TTFC, a transparent thin film inductor (TTFI) and related transparent magnetically coupled devices behave in non-ideal manner, according to researchers, as in [8]. Thus, looking at the benefits, further investigations by the researchers are being continued to realise high-performance TTFI and other transparent magnetically coupled devices.

Transparent thin film transistor (TTFT), the heart of transparent electronics, has ITO, a type of TCO, in place of the metals used as gate, source and drain contact materials. "Compared to organic or polymer transistor materials, these new inorganic oxides have higher mobility, better chemical stability, ease of manufacture, and are physically more robust," said John Wager, a professor of electrical and computer engineering at OSU. They are also hard to scratch, 
resist etching and have a very smooth surface, as in [9]. They are made from low cost, easily available elements, which are environment friendly. TTFT, which are created by researchers at OSU, has the potential of transforming the windshield of a car into a GPS-activated map at the touch of a button, or turning a picture window in the house into a high definition video screen, as in [6]. In Figure 3, the corner of a one-dollar bill is covered with a small square glass plate. It seems that the glass is empty, but, in fact, 56 patterned transistors and 24 resistance test structures cover the glass.

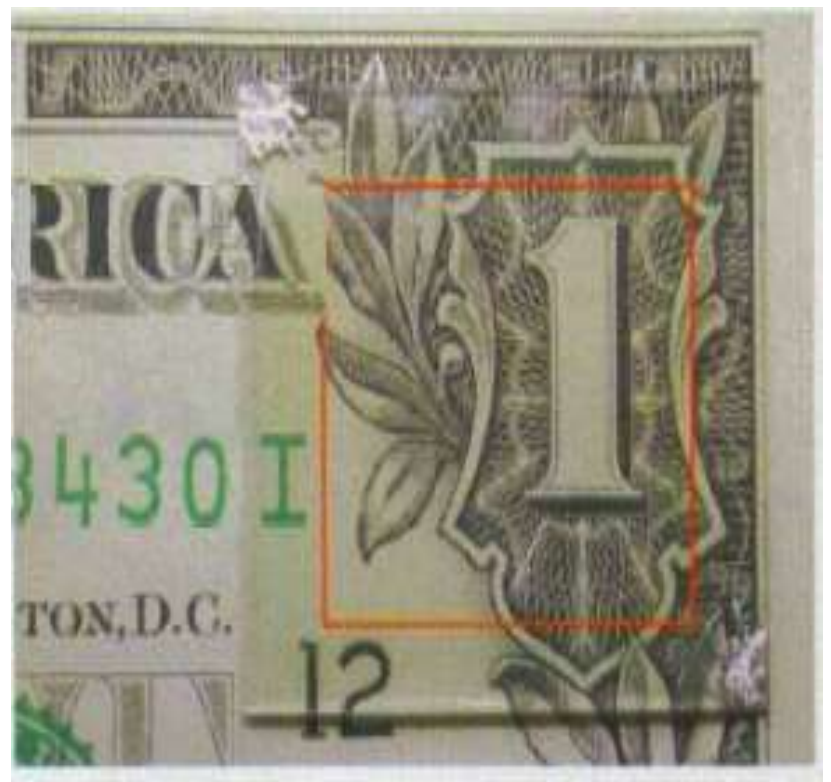

Fig 4: Spin coated ZnO TTFTs. 56 patterned ZnO TTFTs and 24 contact resistance test structures are present inside the orange box, as in [6]

\section{DISCUSSION}

Transparent electronics is about to shake up the field of consumer electronics. It has been possible because the availability of classes of materials for transparent electronics have grown dramatically. Transparent conducting oxides have found their utility in anti-static coating, touch display panels, solar cells, flat panel displays, heaters, defrosters, 'smart windows' and optical coatings, as in [8].

Conceptually, transparent electronics could be incorporated into any glass surface. The interaction between human and electronics would grow upto such a limit that our washroom mirror could display our daily schedule in an updateable way, our drapes could be replaced with a bedroom window that would automatically or manually darken to block out light, as in [10].

\section{FUTURE SCOPE}

There are positive signs showing that the transparent electronics market is beginning to move beyond the products that are already mentioned.
If transparent solar cells are fitted to windows and building facades, then the facades of the house too, harness solar energy to supply the occupants with electrical power, as in [11]. This is what the domestic power supply of the future would look like.

Founders think that the current efficiency, of a glass, coated with a transparent material capable of absorbing infrared and ultraviolet rays, to generate electricity, can be achieved upto $12 \%$, which would be enough to provide one-third of the energy required by a sky-scrapper, as in [1].

Though much progress has been made in developing new materials and devices, there is still plenty of opportunity to study and improve device performance and fabrication techniques.

With the increase in price of gasoline and other exhaustible resources, the field of energy harvesting is gaining height. The increase in environmental pollution, caused by traditional techniques could be controlled only with a marked increase of environment friendly products such as, transparent electronics.

\section{CONCLUSION}

The introduction of transparent conducting oxides has marked a revolutionary phase in the field of electronics. There are lots of potential use, including long term implants, solar cells or environmental sensors, gas sensors and photocatalytic purification of gases and fluids, as in [12].

Transparent electronics technology will be able to emerge out as the best competitor in the commercial product space which is dictated by the multiplicity, performance, cost, manufacturability and reliability of available device types.

Various successful inventions of the researchers in the field of transparent electronics have equipped the world with numerous 'transparent' or 'clear' ways to keep the earth more clean, beautiful and resourceful.

\section{REFERENCES}

[1]. http://www.economictimes.com.

[2]. http://pubs.acs.org.

[3]. Swiss Federal Institute of Technology researchers, "Nature Communications".

[4]. http://www.kaist.edu.

[5]. Transparent electronics.pdf.

[6]. pierceaj@optonline.net.

[7]. http://link.springer.com.

[8]. http://www.wiley.com.

[9]. Advance in Transparent Electronics could spawn new industry, News and Research Communications, OSU.

[10]. New type of semiconductor could change face of consumer electronics.html.

[11]. http://phys.org.

[12].emrs@emrs-strasbourg.com.

[13]. http://ieee.org. 